\title{
REVIEW ARTICLE Drug addiction: a curable mental disorder?
}

\author{
Jian-feng Liu' ${ }^{1}$ and Jun-xu Li ${ }^{1}$
}

Drug addiction is a chronic, relapsing brain disorder. Multiple neural networks in the brain including the reward system (e.g., the mesocorticolimbic system), the anti-reward/stress system (e.g., the extended amygdala), and the central immune system, are involved in the development of drug addiction and relapse after withdrawal from drugs of abuse. Preclinical and clinical studies have demonstrated that it is promising to control drug addiction by pharmacologically targeting the addiction-related systems in the brain. Here we review the pharmacological targets within the dopamine system, glutamate system, trace amine system, antireward system, and central immune system, which are of clinical interests. Furthermore, we discuss other potential therapies, e.g., brain stimulation, behavioral treatments, and therapeutic gene modulation, which could be effective to treat drug addiction. We conclude that, although drug addiction is a complex disorder that involves complicated neural mechanisms and psychological processes, this mental disorder is treatable and may be curable by therapies such as gene modulation in the future.

Keywords: drug addiction; pharmacological targets; behavioral treatment; brain stimulation; gene therapy

Acta Pharmacologica Sinica (2018) 39:1823-1829; https://doi.org/10.1038/s41401-018-0180-x

\section{INTRODUCTION}

Drug addiction is a chronic, relapsing mental disorder characterized by compulsive drug-seeking despite severe negative subsequences [1]. Clinically, drug addiction/substance-use disorder, which is diagnosed by standardized examinations such as the Diagnostic and Statistical Manual of Mental Disorders, Fifth Edition (DSM-V), is distinct with the occasional use of drugs of abuse. To be diagnosed as drug addiction by DSM-V, patients should meet the diagnostic criteria for each drug of abuse, which are defined as mild, moderate, or severe, to indicate the level of severity. In general, drug addiction develops chronically with the following four stages: occasional use, recreational use, regular use, and addiction [2]. Current studies investigating the neural mechanisms of drug addiction has been focusing on compulsive intake when addicted to drugs and relapse after abstinence [1, 3]. Along with the neck-breakingly fast developments of biological research approaches, especially molecular neuroscience, it seems that we have moved into a new era that considerable approaches are promising to be translated into effective clinic tools for treating mental disorders. However, the lost in translation from basic research to clinical treatments is a common challenge in various pathological disorders, including addiction treatments [4].

Pharmacological interventions are the major therapies for treating human diseases. However, similar to other mental disorders, drug addiction is profoundly influenced by emotion, consciousness, and cognition [5]. Besides pharmacological interventions, behavioral strategies are also promising for treating drug addiction. To some extent, non-pharmacological treatments may have greater advantages because of their limited side effects [6]. Here we review potential strategies that we deem most important and promising for treating drug addiction and preventing relapse.

\section{PHARMACOLOGICAL TARGETS}

Current pharmacological treatments of drug addiction primarily target the specific receptors that the drugs of abuse act on [7]. For example, the most successful and recommended treatment for opioid-use disorder is opioid agonist maintenance treatment by using the opioid partial agonist buprenorphine or opioid full agonist methadone $[8,9]$. In addition, the opioid antagonist naltrexone that displaces opioid agonists has been used as a medicine for the treatment of opioid dependence and relapse for decades [10]. Likewise, nicotine addiction is usually treated by modulating the activity of nicotine receptors. Currently, three major pharmaceutics that were approved by United States Food and Drug Administration to treat nicotine addiction are the nicotine receptor antagonist bupropion, the nicotine receptor partial agonist varenicline [11], and nicotine replacement therapy [12]. However, the current medicines cannot effectively prevent the high rate of relapse after abstinence [12]. Furthermore, current treatments could produce serious side effects such as opioid overdose in opioid agonist maintenance treatment [8]. Effective and safer pharmacological treatments are in dire need to treat drug addiction.

\section{Dopaminergic system}

Growing evidence has identified the critical mesolimbic dopaminergic circuit as well as important molecules in this neural pathway that mediate addiction to specific drugs and excessive behaviors [13]. Dopamine, one of the major neurotransmitters in the brain, is believed to be the "culprit" that results in drugs of abuse-induced "high" [14]. As most drugs of abuse eventually activate the dopaminergic system and elevate the dopamine transmission in the brain, modulating the dopamine system could effectively treat most drug addiction, at least in theory [15]. For

\footnotetext{
${ }^{1}$ Department of Pharmacology and Toxicology, University at Buffalo, The State University of New York, Buffalo, NY 14203, USA
}

Correspondence: Jian-feng Liu (jliu66@buffalo.edu) or Jun-xu Li (junxuli@buffalo.edu)

Received: 11 September 2018 Accepted: 29 September 2018

Published online: 31 October 2018 
this reason, many efforts have been made to investigate potential pharmacological targets within the dopaminergic system.

The dopamine system has been discovered for over 60 years [16]. Since then, accumulating evidence has demonstrated a crucial role of the dopamine system in regulating several brain diseases, especially drug addiction, Parkinson's disorder, schizophrenia, anxiety, and depression [16]. The signal of dopamine is mediated by dopamine receptors and several downstream messengers, such as protein kinase $A$, protein kinase $C$, extracellular signal-regulated kinase, $\mathrm{Ca}^{2+}$ /calmodulin-dependent kinase II, and DARPP-32 [14, 17, 18]. Among them, DARPP-32, which is enriched in the dopamine-innervated brain areas, could be a promising candidate for treating drug addiction [15]. As the downstream molecules of dopamine receptors also have common roles in mediating other $\mathrm{G}$ protein-coupled receptor signals [19], activation of these molecules could result in unwanted side effects. Thus, most of the downstream molecules of dopamine receptors are not appropriate therapeutic targets. Pharmacologically targeting the dopamine receptors for treating drug addiction is of great clinical interest. Dopamine receptors in the brain include two main subfamilies: D1-like receptors (D1 and D5) and D2-like receptors (D2, D3, and D4) [20]. Compared with D1 receptors, D2 receptors are more attractive as a candidate drug target, as D2 receptors are preferentially involved in chronic drug exposure and relapse behavior [20]. Other dopamine receptor subtypes, such as D3 receptors, are also promising candidate drug targets for addiction treatment $[7,21]$. Due to the high structural homology between D2 and D3 receptors, compounds that target D3 receptors are usually able to bind D2 receptors. Fortunately, recent studies have developed some D3-selective ligands and these compounds showed potentials as modifiers of drug addiction [22]. It has been shown that GSK598809, a selective D3 receptor antagonist, is efficacious to reduce addiction-related behaviors in preclinical models of relapse [22]. A recent study showed that GSK598809 normalized the reward deficits in drugdependent subjects [22]. It was further demonstrated that GSK598809 might selectively modulate the neural network underlying reward anticipation. More importantly, GSK598809 is currently in clinical trials for treating drug addiction [7].

\section{Glutamate system}

As mentioned above, drug addiction developed after repeated drug exposure is different from occasional use of drugs, which is mainly dependent on the acute effects of drugs [3, 23]. The neuroadaptations induced by chronic drug use involve several important neural systems [23]. Increasing evidence has shown that the glutamatergic system in the ventral striatum/nucleus accumbens (NAC) and the prefrontal cortex (PFC) emerges as the dominant factor that mediates drug-seeking and relapse after abstinence from drugs [24, 25].

Glutamate system in the brain contributes to the adaptive alterations induced by chronic drug exposure [24, 26]. Actions of glutamate are mediated through its receptors, among which the a-amino-3-hydroxy-5-methyl-4-isoxazolepropionic acid receptors (AMPARs), the $\mathrm{N}$-methyl-D-aspartate receptors (NMDARs), and the metabotropic glutamate receptors (mGluRs) are the major glutamate receptors that are involved in drug addiction [24, 27, 28]. Activation of glutamate system in the corticostriatal areas partially contributes to the relapse to continued drug-taking and -seeking [23]. Pharmacological modulations of AMPARs and NMDARs could attenuate abuse-related behaviors of drugs. The interested readers are referred to other excellent reviews for more information on this topic [29-32]. It should be noted that although many compounds that antagonize ionotropic glutamate receptors show efficacy to prevent addictive-like behaviors, most of them are not suitable for clinical use because of their potential side effects. Recently, it was demonstrated that subanesthetic doses of ketamine, a NMDAR antagonist, attenuated cocaine addiction without inducing psychiatric disturbances [33, 34]. Although ketamine has abuse potential per se, these data strongly support that subanestheic ketamine could be used as a novel pharmacotherapy to treat drug addiction. It is especially intriguing that subanesthetic ketamine has been shown to possess rapid antidepressant effects in both preclinical and clinical studies [35-37]. Abundant epidemiological data have revealed high comorbidity of drug addiction and major depression [38]; therefore, the above results suggest that subanesthetic ketamine could be especially effective to treat the comorbidity of drug addiction and depression [39]. Besides directly modulating the ionotropic glutamate receptors, recent studies have demonstrated promising effects for the use of allosteric modulators of mGluRs in drug addiction [40]. Preliminary studies showed that positive and negative allosteric modulators were able to reduce various addictive-like behaviors in animal models [40].

Pharmacological modulation of glutamate lactate transporters (GLTs), which are important for extracellular glutamate clearance, is also a promising strategy for treating drug addiction [41]. GLT-1, also referred as solute carrier family 1 member 2 and excitatory amino acid transporter 2 , is the predominant transporter attributing to glutamate uptake in the brain [42]. Preclinical and clinical studies investigating the role of GLT-1 in drug addiction has been systematically reviewed recently [41]. In general, most preclinical evidence revealed that reversal of the GLT-1 dysfunction that was induced by drug exposure could effectively suppress drug-seeking behavior [43]. There are several clinical trials that investigated the efficacy of $\mathrm{N}$-acetylcysteine (NAC), a drug regulating GLT-1, for treating several drugs of abuse, such as cocaine, methamphetamine, cannabis, and nicotine [44]. However, the clinical results are mixed. For example, it was reported that NAC reduced self-reported cocaine use in a small trial [45]. However, in a large double-blind placebo-controlled study, it was shown that NAC was effective to prolong the time to relapse but had no effect on self-reported cocaine use [46]. A study by using the proton magnetic resonance spectroscopy showed that NAC reduced the elevated glutamate levels in the dorsal anterior cingulate cortex in cocaine-dependent patients, suggesting that the inhibitory effects of NAC were partially due to its ability to normalize the abnormal glutamate transmission [47]. Recently, it is demonstrated that the NAC derivative NAC-amide, which has better bioavailability than NAC, was also able to attenuate cocaine-seeking behaviors, suggesting a potential use or even greater efficacy of NAC-amide in clinical settings [48, 49]. It should be kept in mind that NAC and NAC-amide are antioxidant and anti-stress molecules [50]. The effects of these compounds on addiction are possibly due to their anti-stress properties, for the stress/anti-reward system also has a critical role in drug addiction [51].

\section{Trace amine system}

Trace amines are a group of amines that are structurally similar to classic amino amines such as dopamine $[52,53]$. Compared with the extensive accumulation of classic amines in the brain, the concentrations of endogenous trace amines are relatively low (nanomolar) [53]. A family of trace amine-associated receptors (TAARs) have been cloned in 2001 [53]. Among them, TAAR1 is the best-studied receptor of trace amines [54]. Recent studies have revealed that TAAR1 participates in several mental disorders including depression, schizophrenia, and drug addiction [52].

TAAR1 is broadly expressed in the brain with a relative high accumulation in the mesocoticolimbic system [52]. It is demonstrated that modulation of TAAR1 could regulate dopamine transmission, indicating a possible role of TAAR1 in dopaminerelated behaviors [54]. Studies from our lab and others have shown that TAAR1 agonists were effective to attenuate the behavioral and biochemical effects of stimulants such as cocaine and amphetamines [52]. Our recent study also demonstrated that 
activation of TAAR1 was effective to reduce nicotine-induced dopamine release and neural activation in the NAc [55]. Furthermore, TAAR1 agonists attenuated several kinds of nicotine-related behaviors, e.g., nicotine-induced behavioral sensitization, nicotine discrimination, nicotine self-administration, and reinstatement of nicotine-seeking [55]. Furthermore, we demonstrated that the dosages of TAAR1 agonists that effectively inhibited addiction-related behaviors had no effect on general motor activity [55]. These results indicated that modulation of TAAR1 could selectively regulate abuse-related behaviors of several drugs of abuse. Currently, several compounds that modulate the activity of TAAR1 are in clinical trials designed to treat schizophrenia. Nevertheless, although TAAR1 is a promising target for addiction treatment, the function and the neural mechanism of TAAR1 are still poorly understood [52]. Future studies are needed to determine the exact neural mechanisms of TAAR1 in regulating drug addiction.

\section{Stress/anti-reward system}

Besides the positive reinforcing effects of drug use, e.g., euphoria or "high," negative reinforcing effects associated with abstinence are also well-known factors that contribute to the development of drug addiction [2,56]. The negative reinforcement of drugs recruits a brain system that is opposed to the rewarding system, termed the anti-reward/stress system in the brain $[2,57]$. It is believed that the neuroanatomical substrate of the anti-reward system is the extended amygdala that includes the bed nucleus of the stria terminalis, the central amygdala, and the NAc shell $[2,51]$. These brain regions were demonstrated to participate in both the physical withdrawal symptoms and negative emotional states that drive compulsive drug use, drug-seeking, and relapse [51, 58]. As mentioned above, the comorbidity of addiction and other mental disorders are common in patients with substance-use disorder. Drug use- and withdrawal-induced emotional alterations may interact with or facilitate the occurrence of other mental disorders, which in turn promote drug use and relapse $[59,60]$.

Several important molecular targets in the anti-reward system have been studied [3]. Among them, corticotropin-releasing factor (CRF) is one of the best-studied molecules [3]. A very large number of studies have verified the role of CRF in stress-related behaviors and drug-seeking behavior associated with major drugs of abuse. Preclinical studies demonstrated that CRF peptide and receptor antagonists prevented anxiety-like responses and aversive-like motivational effects induced by withdrawal from several drugs of abuse, as well as attenuated the increased self-administration of drugs in the rat extended-access model of drug intake [51]. Recently, a clinical study investigated the effects of CRF1 antagonist verucerfont on alcohol addiction [61]. Unfortunately, although verucerfont was able to prevent the hypothalamicpituitary-adrenal axis response, it did not affect stress-induced alcohol craving [61]. Nevertheless, it is too early to conclude that targeting the CRF system to treat drug addiction is deemed to fail, given the very strong preclinical evidence to suggest otherwise [51]. More carefully designed clinical trials are required to test the translational possibility of other CRF antagonists from animals to humans.

\section{Immune system}

Recent evidence has revealed a critical role of the neuroimmune system in psychiatric disorders including drug addiction [62, 63]. The central immune system is composed of multiple types of cells, cytokines, and intracellular signaling pathways in the brain [64]. Some studies demonstrated that drugs of abuse, such as opioids, cocaine, methamphetamine, and ethanol, can activate central immune signaling, which in turn enhances the reinforcing effects of drugs [65]. However, the role of immune system in drug addiction remains unclear and other studies also reported no effect of immune function modulators on addition-related behaviors [62]. Furthermore, although a growing number of immune-related targets, such as tumor necrosis factor-a, interleukin-1 $\beta$ (IL-1 $\beta)$, and IL-6, have been demonstrated to have important roles in regulating drug addiction, only a few of them are pharmacologically significant [62]. Here we will focus on one immune-related target, the Toll-like receptor 4 (TLR4), which we think is a promising pharmacological candidate for treating drug addiction [66].

TLR4 is a member of TLR family, which has a key role in the innate immune system [66]. Compared with other drugs of abuse, opioids are the best-studied drugs that could be regulated by TLR4. Opioids could directly activate the TLR4 to produce a neuroinflammatory response [67]. A growing number of studies revealed that inhibition of TLR4 suppressed morphine tolerance and the rewarding effects of morphine, and enhanced the analgesic properties of morphine $[68,69]$. A recent study showed that chronic but not acute delivery of the TLR4 antagonist $(+)$-naltrexone reduced the incubation of cue-induced heroinseeking [70]. It was demonstrated that TLR4 is also involved in cocaine addiction. A study showed that TLR4 in the NAc mediates the reinforcing properties of cocaine [71]. In addition, activation of TLR4 in the ventral tegmental area (VTA) was sufficient to reinstate distinguished cocaine-seeking behavior [72]. Another study also reported that TLR4 mediated methamphetamine-induced neuroinflammatory response in astrocytes [73]. However, discrepant results were also reported. In a study, the dosages of $(+)$-naltrexone that significantly inhibited remifentanil and cocaine self-administration produced an inhibitory effect on the rate of food-maintained responding, indicating a nonspecific inhibition of general activity rather than a specific anti-reward effect [74]. Moreover, ( + )-naltrexone had no effect on heroin selfadministration and the incubation of cue-induced methamphetamine-seeking [70]. Taken together, the role of TLR4 in drug addiction remains somewhat elusive, which may be dependent on the types of drugs, treatment regimens, and experimental conditions.

Currently, there is no clinical evidence that modulation of central immune system is effective for treating drug addiction. As immune response is probably the most important biological reactions of the human body in responding to environmental and innate stimuli, it is a challenge to selectively inhibit the hyperactivity of central immune system induced by repeated drug exposure, while leaving the peripheral immune system intact.

\section{BRAIN STIMULATION}

Considerable evidence has demonstrated that modulating the activities of addiction-related brain areas is a promising strategy to control drug use and relapse [75]. Although there are various ways to modulate the activity of a specific brain area, such as random noise stimulation, ultrasound stimulation, electoral stimulation, and magnetic stimulation, recent research has been focusing on the latter two [76, 77]. Transcranial magnetic stimulation (TMS) and transcranial direct current stimulation (tDCS) are two of the best-studied approaches for treating drug addiction and other mental disorders [76-79]. Studies have shown that stimulating dorsal lateral PFC by TMS and tDCS were effective to reduce craving, improve cognition, and ameliorate the mood states in subjects using major drugs of abuse $[76,80]$. Importantly, TMS and tDCS showed greater advantages than other invasive strategies including deep brain stimulation (DBS) that requires invasive electrodes to be implanted into the target brain areas [80]. It should be noted that, although TMS and tDCS are relatively safer than invasive stimulations, several disadvantages limit their application in the clinic. For example, (1) these techniques could inaccurately stimulate other nearby brain areas; (2) they cannot reach deeper brain areas that could be easily achieved by DBS; (3) 
an inaccurate manipulation could induce unwanted effects such as seizures [81]. The detailed technological issues of these approaches are beyond the scope of this review and interested readers can find more information elsewhere [81, 82].

Recent preclinical studies have developed and utilized virusmediated strategies to modulate the activities of brain areas [83]. Extensive evidence has demonstrated that chemogenetic and optogenetic modulations of reward and anti-reward systems, including the NAc, the PFC, the VTA, and the amygdala, were able to reduce drug-taking and-seeking [84]. These techniques by and large reconfirmed previous conclusions that were achieved by pharmacological interventions, as well as provided novel insights that may shed light on new neural mechanisms [84]. Chemogenetic and optogenetic approaches could easily target a specific circuit rather than just a specific brain area. Stimulation of a specific area could generally alter the activities of its projecting areas, which could induce unwanted side effects [85]. For example, stimulation of the PFC could affect activities of the NAc, hippocampus, and amygdala [86]. Because of the important roles of the hippocampus and amygdala in regulating memories and emotions, stimulating the PFC would also alter subjects' emotional states and cause amnesia. Current approaches are impossible to specifically modulate a neural circuit in humans. Nevertheless, results from animal studies could provide us with clues of novel neural mechanisms and possible side effects induced by brain stimulation in humans, which in turn help us to adjust and optimize stimulation parameters. For example, a recent study showed that mimicking optogenetic stimulation-induced normalization of synaptic transmission in the NAc by the combination of DBS and pharmacological intervention persistently reduced cocaine-induced sensitization [87]. The study proved the validity of combining brain stimulation with other treatments such as drugs and cognitive behavioral treatments to treat drug addiction.

Accordingly, more studies are required by using optogenetic and chemogenetic approaches to identify novel brain areas that participate in drug addiction. It should be noted that neural circuit-related studies in animals are probably not translable to humans due to the great heterogeneity of brain anatomy between rodents and humans [88]. In addition, current techniques only allow us to stimulate one brain region at one time, which would limit the efficacy of brain stimulation. As mentioned above, a broad range of brain areas synergistically contribute to drug addiction. If we could develop novel techniques that allow us to simultaneously stimulate addiction-related brain areas, stronger and long-lasting effects could be expected.

\section{BEHAVIORAL TREATMENT}

Behavioral approaches, such as cognitive behavioral therapy (CBT) and cue exposure therapy (CET) are effective for treating drug addiction $[89,90]$. Drug addiction is a unique mental disorder in humans. Executive functions, especially the higher-order executive functions such as inhibitory control and cognitive flexibility, have a fundamental role in coping with the mood disturbances and incubated craving associated with protracted withdrawal from drugs of abuse [91]. The potential of CBT for treating drug addiction is based on the hypothesis that reversal of the maladaptive information processing and abrupt beliefs associated with drug addiction is able to reshape the addiction-related behaviors. CBT has been shown to be effective in reducing drug craving in clinical settings when applied alone or with other pharmacotherapy [89]. Furthermore, CBT is widely applicable across many psychiatry fields and has been implemented in a broad range of psychiatric disorders [92]. However, implementation of CBT for treating drug addiction is currently uncommon. This could be due to several disadvantages of CBT, including relatively high cost and requirement of physician specialists to apply the therapy [93]. Furthermore, although CBT shows a timelimit advantage compared with traditional talk therapy, additional treatment cycles are required to produce persistent therapeutic efficacy, otherwise patients would generally relapse within months. The computer-assisted CBT may be an alternative option, which has revealed significant advantages compared with the traditional CBT [94]. For example, computer-assisted CBT is low cost and in no need of clinicians with special skills. The acceptance and accessibility of computer-assisted CBT are growing due to the increasing access to computer and internet. Studies have shown that a six-module computer-based training in CBT (CBT4CBT) was an effective adjunct to standard drug addiction treatments [94, 95].

CET is another behavioral strategy that was widely accepted within drug addiction field and throughout psychiatry [90]. The premise of CET is that Pavlovian conditioned learning contributes to cue-induced craving and drug-seeking, and repeated exposure to drug-associated cues (contextual and discrete cues), which is called extinction, will eventually lead to a decrease in conditioned responses, e.g., cue-induced drug-seeking and craving. CET is based on the hypothesis that drug addiction is an abrupt memory that usurps the normal learning and memory system in the brain [6]. However, in contrast to the positive reports in other psychiatric disorders (e.g., anxiety disorders), studies have not shown a positive efficacy of CET for treating drug addiction in clinical settings. Another psychological process reconsolidation, a state that is triggered by memory retrieval and reactivation, is usually mentioned and discussed with extinction in the literature [96]. Modulation of reconsolidation and extinction of drug reward memory with either pharmacological or behavioral strategies have been demonstrated to be effective for treating addiction. We have reviewed the advances in this topic in a recent review [6]. It should be noted that the lack of efficacy of CET in humans may imply that targeting the cue-associated memories probably only has limited effects on addiction-related behaviors [90]. This could be due to that interventions on drug reward memory would effectively eliminate the cue-induced responses, while leaving the druginduced maladaptive plasticity alterations intact. In addition, craving induced by drug-associated cues may not be the fuse triggering relapse. With this in mind, future research is warranted to address whether the modulation of drug reward memory could reverse the maladaptive behavioral and neural elasticities, and then develop novel memory-related strategies for treating addiction.

\section{THERAPEUTIC GENE MODULATION}

The long-lasting neuroplasticity changes induced by exposure to drugs of abuse are mediated by specific gene transcriptions [97]. Genetic analysis and functional studies have identified a wide range of candidate genes and their variants that regulate drug addiction, e.g., dopamine-related genes, glutamate-related genes, and transcription factors (e.g., the immediate early gene fosB) [9799]. It has been shown that the development of addiction is positively associated with genetic factors, suggesting a high heritability feature of addiction [100]. Drug addiction is not heritable across generations, but personality traits that are associated with initiation of drug use are heritable [100]. Furthermore, epigenetic alterations and epigenetic regulators, e.g., chromatin-remodeling enzymes, histone acetyltransferases, and methyltransferases, also have critical roles in mediating the long-lasting effects of drug use [101]. Recent studies also found that microRNAs and other non-coding RNAs are also important factors that mediate rewarding properties of drugs of abuse, suggesting that modulation of posttranscriptional RNAs may be a possible pharmacotherapy to reverse drug-induced neuroplasticity in the brain $[102,103]$. Taken together, current evidence indicates that therapeutic gene modulation altering the 
addiction-related genes and/or their expression patterns would be a promising therapy for treating drug addiction.

Gene therapy is usually achieved by delivering a variety of types of plasmids and vectors carrying therapeutic genes into human tissues. A recently developed technology known as CRISPR-Cas9 has been shown to be able to effectively alter genes in vivo [104]. Importantly, studies have revealed that CRISPR-Cas9 can highly selectively alter the target gene and do not cause off-target effects. Recently, a study published last year showing "unexpected mutations after CRISPR-Cas9 editing in vivo" has been retracted because of serious flaws of the experimental design [105]. Intriguingly, clinical trials are about to begin to test the validity of CRISPR-Cas9 in treating human diseases. Although current gene therapy is only used to treat cancer, genetic diseases, and infectious diseases [106], preclinical studies by using virusmediated expression or knockdown of addiction-related genes have been revealed to be effective in altering drug-induced neuroplasticity and maladaptive behaviors [107]. For example, studies showed that helper-dependent adenoviral vector or adeno-associated virus vector-mediated expression of a butyrylcholinesterase-based cocaine hydrolase, which has great efficacy in metabolizing cocaine, was effective to block cocaine's behavioral effects and relapse in animal models [108, 109]. We believe that it is about time to pay more serious attention to gene therapy in treating drug addiction. More importantly, therapeutic gene modulation might be possible to achieve true cure for drug addiction, because the results of gene therapy are usually longlasting if not permanent.

\section{CONCLUSION}

Drug addiction is a complicated mental disorder, because multiple levels of biological and psychological systems are recruited into its development. Studies have demonstrated that the reward system (e.g., the mesocorticolimbic system) and the anti-reward system (e.g., the extended amygdala) synergistically contribute to relapse after protracted withdrawal. Besides the current treatments that target specific receptors, pharmacological approaches that target the molecular candidates in the reward and anti-reward systems is promising to treat drug addiction. The neuroimmune system is a relatively novel target that is of significant clinical interest. However, the role of neuroimmune system in drug addiction remains poorly understudied. Other strategies, such as brain stimulation and behavioral treatments, may have greater advantages than pharmacotherapies due to their safer profiles. Overall, the clinical efficacy of current treatments of drug addiction is limited and relapse remains a major clinical challenge. In regards to achieving long-term treatment success and possibly permanent cure, gene therapy could be a powerful strategy, despite that this approach remains in its infancy in the treatment of drug addiction.

\section{ACKNOWLEDGEMENTS}

This work was supported by the National Institutes of Health National Institute on Drug Abuse [Grant R21DA040777]. The content is solely the responsibility of the authors and does not necessarily represent the official views of the National Institutes of Health.

\section{ADDITIONAL INFORMATION}

Competing interests: The authors declare no competing interests.

\section{REFERENCES}

1. Nestler EJ. Molecular basis of long-term plasticity underlying addiction. Nat Rev Neurosci. 2001;2:119-28.

2. Koob GF. Antireward, compulsivity, and addiction: seminal contributions of Dr. Athina Markou to motivational dysregulation in addiction. Psychopharmacology (Berl). 2017;234:1315-32
3. Koob GF. Addiction is a reward deficit and stress surfeit disorder. Front Psychiatry. 2013;4:72.

4. Fuehrlein BS, Ross DA. Opioid use disorder: a desperate need for novel treatments. Biol Psychiatry. 2017;81:e43-e5.

5. Kosarov D. Vectorelectromyographic investigations at high muscle activity. Electromyogr Clin Neurophysiol. 1975;15:453-73.

6. Liu JF, Tian J, Li JX. Modulating reconsolidation and extinction to regulate drug reward memory. Eur J Neurosci. 2018. https://doi.org/10.1111/ejn.14072.

7. Newman AH, Blaylock BL, Nader MA, Bergman J, Sibley DR, Skolnick P. Medication discovery for addiction: translating the dopamine D3 receptor hypothesis. Biochem Pharmacol. 2012;84:882-90.

8. Bonhomme J, Shim RS, Gooden R, Tyus D, Rust G. Opioid addiction and abuse in primary care practice: a comparison of methadone and buprenorphine as treatment options. J Natl Med Assoc. 2012;104:342-50.

9. Jiang $\mathrm{H}$, Hillhouse $\mathrm{M}$, Du J, Pan S, Alfonso A, Wang J, et al. Dose, plasma level, and treatment outcome among methadone patients in Shanghai, China. Neurosci Bull. 2016;32:538-44.

10. Larney S, Gowing L, Mattick RP, Farrell M, Hall W, Degenhardt L. A systematic review and meta-analysis of naltrexone implants for the treatment of opioid dependence. Drug Alcohol Rev. 2014;33:115-28.

11. Ebbert JO, Hughes JR, West RJ, Rennard SI, Russ C, McRae TD, et al. Effect of varenicline on smoking cessation through smoking reduction: a randomized clinical trial. JAMA. 2015;313:687-94.

12. Unauthored. Nicotine Replacement Therapy for Smoking Cessation or Reduction: A Review of the Clinical Evidence. Canadian Agency for Drugs and Technologies in Health; 2014 Jan 16.(Ottawa (ON), 2014).

13. Luscher $C$. The emergence of a circuit model for addiction. Annu Rev Neurosci. 2016;39:257-76.

14. Baik JH. Dopamine signaling in reward-related behaviors. Front Neural Circuits. 2013;7:152.

15. Nishi A, Shuto T. Potential for targeting dopamine/DARPP-32 signaling in neuropsychiatric and neurodegenerative disorders. Expert Opin Ther Targets. 2017;21:259-72.

16. Iversen SD, Iversen LL. Dopamine: 50 years in perspective. Trends Neurosci. 2007;30:188-93.

17. Wang B, Yang X, Sun A, Xu L, Wang S, Lin W, et al. Extracellular signal-regulated kinase in nucleus accumbens mediates propofol self-administration in rats. Neurosci Bull. 2016;32:531-7.

18. Cui SZ, Wang SJ, Li J, Xie GQ, Zhou R, Chen L, et al. Alteration of synaptic plasticity in rat dorsal striatum induced by chronic ethanol intake and withdrawal via ERK pathway. Acta Pharmacol Sin. 2011;32:175-81.

19. Stefan $E$, Malleshaiah MK, Breton B, Ear PH, Bachmann V, Beyermann $M$, et al. PKA regulatory subunits mediate synergy among conserved $G$-protein-coupled receptor cascades. Nat Commun. 2011;2:598.

20. Volkow ND, Fowler JS, Wang GJ, Baler R, Telang F. Imaging dopamine's role in drug abuse and addiction. Neuropharmacology. 2009;56(Suppl 1):3-8.

21. Sun L, Song R, Chen Y, Yang RF, Wu N, Su RB, et al. A selective D3 receptor antagonist YQA14 attenuates methamphetamine-induced behavioral sensitization and conditioned place preference in mice. Acta Pharmacol Sin. 2016;37:157-65.

22. Murphy A, Nestor LJ, McGonigle J, Paterson L, Boyapati V, Ersche KD, et al. Acute D3 antagonist GSK598809 selectively enhances neural response during monetary reward anticipation in drug and alcohol dependence. Neuropsychopharmacology. 2017;42:1049-57.

23. Pierce RC, Wolf ME. Psychostimulant-induced neuroadaptations in nucleus accumbens AMPA receptor transmission. Cold Spring Harb Perspect Med. 2013;3:a012021.

24. Knackstedt LA, Kalivas PW. Glutamate and reinstatement. Curr Opin Pharmacol. 2009:9:59-64.

25. Yang JY, Qi J, Han WY, Wang F, Wu CF. Inhibitory role of oxytocin in psychostimulant-induced psychological dependence and its effects on dopaminergic and glutaminergic transmission. Acta Pharmacol Sin. 2010;31:1071-4.

26. Wang XF, Zhao TY, Su RB, Wu N, Li J. Agmatine prevents adaptation of the hippocampal glutamate system in chronic morphine-treated rats. Neurosci Bull. 2016;32:523-30.

27. Zhu H, Lai M, Chen W, Mei D, Zhang F, Liu H, et al. N-acetylaspartylglutamate inhibits heroin self-administration and heroin-seeking behaviors induced by cue or priming in rats. Neurosci Bull. 2017;33:396-404.

28. Lou ZZ, Chen LH, Liu HF, Ruan LM, Zhou WH. Blockade of mGluR5 in the nucleus accumbens shell but not core attenuates heroin seeking behavior in rats. Acta Pharmacol Sin. 2014;35:1485-92.

29. Kalivas PW, Volkow ND. New medications for drug addiction hiding in glutamatergic neuroplasticity. Mol Psychiatry. 2011;16:974-86.

30. Wolf ME. Synaptic mechanisms underlying persistent cocaine craving. Nat Rev Neurosci. 2016;17:351-65. 
31. Huang $X$, Ni W, Zhang C. Calcium-impermeable NMDA receptor: a novel target for addiction. Neurosci Bull. 2017;33:357-8.

32. Lin XJ, Zhang JJ, Yu LC. GluR2-3Y inhibits the acquisition and reinstatement of morphine-induced conditioned place preference in rats. Neurosci Bull. 2016;32:177-82.

33. Dakwar E, Levin F, Foltin RW, Nunes EV, Hart CL. The effects of subanesthetic ketamine infusions on motivation to quit and cue-induced craving in cocainedependent research volunteers. Biol Psychiatry. 2014;76:40-6.

34. Dakwar E, Hart CL, Levin FR, Nunes EV, Foltin RW. Cocaine self-administration disrupted by the $\mathrm{N}$-methyl-D-aspartate receptor antagonist ketamine: a randomized, crossover trial. Mol Psychiatry. 2017;22:76-81.

35. Li N, Lee B, Liu RJ, Banasr M, Dwyer JM, Iwata M, et al. mTOR-dependent synapse formation underlies the rapid antidepressant effects of NMDA antagonists. Science. 2010;329:959-64

36. Duman RS, Aghajanian GK. Synaptic dysfunction in depression: potential therapeutic targets. Science. 2012;338:68-72.

37. Zhu W, Ding Z, Zhang Y, Shi J, Hashimoto K, Lu L. Risks associated with misuse of ketamine as a rapid-acting antidepressant. Neurosci Bull. 2016;32:557-64.

38. $\mathrm{Ng} \mathrm{E}$, Browne CJ, Samsom JN, Wong AHC. Depression and substance use comorbidity: what we have learned from animal studies. Am J Drug Alcohol Abus. 2017;43:456-74.

39. Yuan K, Han Y, Hashimoto K, Lu L. On the eve of upgrading antidepressants: (R)ketamine and its metabolites. Neurosci Bull. 2016;32:565-8.

40. Olive MF. Metabotropic glutamate receptor ligands as potential therapeutics for addiction. Curr Drug Abus Rev. 2009;2:83-98.

41. Spencer S, Kalivas PW. Glutamate transport: a new bench to bedside mechanism for treating drug abuse. Int J Neuropsychopharmacol. 2017;20:797-812.

42. Rimmele TS, Rosenberg PA. GLT-1: the elusive presynaptic glutamate transporter. Neurochem Int. 2016;98:19-28.

43. Roberts-Wolfe DJ, Kalivas PW. Glutamate transporter GLT-1 as a therapeutic target for substance use disorders. CNS Neurol Disord Drug Targets. 2015;14:745-56.

44. Nocito Echevarria MA, Andrade Reis T, Ruffo Capatti G, Siciliano Soares V, da Silveira DX, Fidalgo TM. N-acetylcysteine for treating cocaine addiction - a systematic review. Psychiatry Res. 2017;251:197-203.

45. Mardikian PN, LaRowe SD, Hedden S, Kalivas PW, Malcolm RJ. An open-label trial of $\mathrm{N}$-acetylcysteine for the treatment of cocaine dependence: a pilot study. Prog Neuropsychopharmacol Biol Psychiatry. 2007;31:389-94.

46. LaRowe SD, Kalivas PW, Nicholas JS, Randall PK, Mardikian PN, Malcolm RJ. A double-blind placebo-controlled trial of $\mathrm{N}$-acetylcysteine in the treatment of cocaine dependence. Am J Addict. 2013;22:443-52.

47. Schmaal L, Veltman DJ, Nederveen A, van den Brink W, Goudriaan AE. Nacetylcysteine normalizes glutamate levels in cocaine-dependent patients: a randomized crossover magnetic resonance spectroscopy study. Neuropsychopharmacology. 2012;37:2143-52.

48. Jastrzebska J, Frankowska M, Filip M, Atlas D. N-acetylcysteine amide (AD4) reduces cocaine-induced reinstatement. Psychopharmacol (Berl). 2016;233:3437-48.

49. Sunitha K, Hemshekhar M, Thushara RM, Santhosh MS, Yariswamy M, Kemparaju $\mathrm{K}$, et al. $\mathrm{N}$-acetylcysteine amide: a derivative to fulfill the promises of $\mathrm{N}$ acetylcysteine. Free Radic Res. 2013;47:357-67.

50. Rushworth GF, Megson IL. Existing and potential therapeutic uses for N-acetylcysteine: the need for conversion to intracellular glutathione for antioxidant benefits. Pharmacol Ther. 2014;141:150-9.

51. Koob GF, Buck CL, Cohen A, Edwards S, Park PE, Schlosburg JE, et al. Addiction as a stress surfeit disorder. Neuropharmacology. 2014;76 Pt B:370-82.

52. Liu JF, Li JX. TAAR1 in addiction: looking beyond the tip of the iceberg. Front Pharmacol. 2018:9:279.

53. Grandy DK. Trace amine-associated receptor 1-Family archetype or iconoclast? Pharmacol Ther. 2007;116:355-90.

54. Pei Y, Asif-Malik A, Canales JJ. Trace amines and the trace amine-associated receptor 1: pharmacology, neurochemistry, and clinical implications. Front Neurosci. 2016;10:148.

55. Liu JF, Seaman R, Jr., Siemian JN, Bhimani R, Johnson B, Zhang Y, et al. Role of trace amine-associated receptor 1 in nicotine's behavioral and neurochemical effects. Neuropsychopharmacology. 2018. https://doi.org/10.1038/s41386-0180017-9

56. Ju YY, Long JD, Liu Y, Liu JG. Formation of aversive memories associated with conditioned drug withdrawal requires BDNF expression in the amygdala in acute morphine-dependent rats. Acta Pharmacol Sin. 2015;36:1437-43.

57. Han Y, Lu L. The other face of the nucleus accumbens: aversion. Neurosci Bull. 2016;32:569-71.

58. Wang J, Wang YH, Hou YY, Xi T, Liu Y, Liu JG. The small GTPase RhoA, but not Rac1, is essential for conditioned aversive memory formation through regulation of actin rearrangements in rat dorsal hippocampus. Acta Pharmacol Sin. 2013;34:811-8.
59. Hamilton KL, Harris AC, Gewirtz JC. Affective and neuroendocrine effects of withdrawal from chronic, long-acting opiate administration. Brain Res. 2013; 1538:73-82.

60. Gong YX, Shou WT, Feng B, Zhang WP, Wang HJ, Ohtsu H, et al. Ameliorating effect of histamine on impairment of cued fear extinction induced by morphine withdrawal in histidine decarboxylase gene knockout mice. Acta Pharmacol Sin. 2010;31:1431-7.

61. Schwandt ML, Cortes CR, Kwako LE, George DT, Momenan R, Sinha R, et al. The CRF1 antagonist verucerfont in anxious alcohol-dependent women: translation of neuroendocrine, but not of anti-craving effects. Neuropsychopharmacology. 2016:41:2818-29.

62. Bachtell RK, Jones JD, Heinzerling KG, Beardsley PM, Comer SD. Glial and neuroinflammatory targets for treating substance use disorders. Drug Alcohol Depend. 2017;180:156-70.

63. Leff-Gelman P, Mancilla-Herrera I, Flores-Ramos M, Cruz-Fuentes C, ReyesGrajeda JP, Garcia-Cuetara Mdel P, et al. The immune system and the role of inflammation in perinatal depression. Neurosci Bull. 2016;32:398-420.

64. Jones KA, Thomsen $C$. The role of the innate immune system in psychiatric disorders. Mol Cell Neurosci. 2013;53:52-62.

65. Jacobsen JH, Hutchinson MR, Mustafa S. Drug addiction: targeting dynamic neuroimmune receptor interactions as a potential therapeutic strategy. Curr Opin Pharmacol. 2016;26:131-7.

66. Hutchinson MR, Northcutt AL, Hiranita T, Wang X, Lewis SS, Thomas J, et al. Opioid activation of toll-like receptor 4 contributes to drug reinforcement. J Neurosci. 2012;32:11187-200.

67. Wang X, Loram LC, Ramos K, de Jesus AJ, Thomas J, Cheng K, et al. Morphine activates neuroinflammation in a manner parallel to endotoxin. Proc Natl Acad Sci USA. 2012;109:6325-30.

68. Eidson LN, Murphy AZ. Blockade of Toll-like receptor 4 attenuates morphine tolerance and facilitates the pain relieving properties of morphine. The. J Neurosci. 2013;33:15952-63.

69. Hutchinson MR, Shavit Y, Grace PM, Rice KC, Maier SF, Watkins LR. Exploring the neuroimmunopharmacology of opioids: an integrative review of mechanisms of central immune signaling and their implications for opioid analgesia. Pharmacol Rev. 2011;63:772-810.

70. Theberge FR, Li X, Kambhampati S, Pickens CL, St Laurent R, Bossert JM, et al. Effect of chronic delivery of the Toll-like receptor 4 antagonist (+)-naltrexone on incubation of heroin craving. Biol Psychiatry. 2013;73:729-37.

71. Kashima DT, Grueter BA. Toll-like receptor 4 deficiency alters nucleus accumbens synaptic physiology and drug reward behavior. Proc Natl Acad Sci USA. 2017;114:8865-70.

72. Brown KT, Levis SC, O'Neill CE, Northcutt AL, Fabisiak TJ, Watkins LR, et al. Innate immune signaling in the ventral tegmental area contributes to drug-primed reinstatement of cocaine seeking. Brain Behav Immun. 2018;67:130-8.

73. Du SH, Qiao DF, Chen CX, Chen S, Liu C, Lin Z, et al. Toll-like receptor 4 mediates methamphetamine-induced neuroinflammation through caspase-11 signaling pathway in astrocytes. Front Mol Neurosci. 2017;10:409.

74. Tanda G, Mereu M, Hiranita T, Quarterman JC, Coggiano M, Katz JL. Lack of specific involvement of (+)-Naloxone and $(+)$-Naltrexone on the reinforcing and neurochemical effects of cocaine and opioids. Neuropsychopharmacology. 2016:41:2772-81.

75. Salling MC, Martinez D. Brain stimulation in addiction. Neuropsychopharmacology. 2016;41:2798-809.

76. Lupi M, Martinotti G, Santacroce R, Cinosi E, Carlucci M, Marini S, et al. Transcranial direct current stimulation in substance use disorders: a systematic review of scientific literature. J ECT. 2017;33:203-9.

77. Hanlon CA, Dowdle LT, Henderson JS. Modulating neural circuits with transcranial magnetic stimulation: implications for addiction treatment development. Pharmacol Rev. 2018;70:661-83.

78. Yang LZ, Yang Z, Zhang X. Non-invasive brain stimulation for the treatment of nicotine addiction: potential challenges. Neurosci Bull. 2016;32:550-6.

79. Akhtar H, Bukhari F, Nazir M, Anwar MN, Shahzad A. Therapeutic efficacy of neurostimulation for depression: techniques, current modalities, and future challenges. Neurosci Bull. 2016;32:115-26.

80. Coles AS, Kozak K, George TP. A review of brain stimulation methods to treat substance use disorders. Am J Addict. 2018;27:71-91.

81. Zhao H, Qiao L, Fan D, Zhang S, Turel O, Li Y, et al. Modulation of brain activity with noninvasive transcranial direct current stimulation (tDCS): clinical applications and safety concerns. Front Psychol. 2017;8:685.

82. Dunlop K, Hanlon CA, Downar J. Noninvasive brain stimulation treatments for addiction and major depression. Ann N Y Acad Sci. 2017;1394:31-54.

83. Deisseroth K. Optogenetics: 10 years of microbial opsins in neuroscience. Nat Neurosci. 2015;18:1213-25.

84. Dong Y, Taylor JR, Wolf ME, Shaham Y. Circuit and synaptic plasticity mechanisms of drug relapse. J Neurosci. 2017;37:10867-76. 
85. Britt JP, Bonci A. Optogenetic interrogations of the neural circuits underlying addiction. Curr Opin Neurobiol. 2013;23:539-45.

86. Klenowski PM. Emerging role for the medial prefrontal cortex in alcohol-seeking behaviors. Addict Behav. 2018;77:102-6.

87. Creed M, Pascoli VJ, Luscher C. Addiction therapy. Refining deep brain stimulation to emulate optogenetic treatment of synaptic pathology. Science. 2015;347:659-64.

88. Kruepunga N, Hakvoort TBM, Hikspoors J, Kohler SE, Lamers WH. Anatomy of rodent and human livers: what are the differences? Biochim Biophys Acta Mol Basis Dis. 2018;pii: S0925-4439:30189-3.

89. Zilverstand A, Parvaz MA, Moeller SJ, Goldstein RZ. Cognitive interventions for addiction medicine: understanding the underlying neurobiological mechanisms. Prog Brain Res. 2016;224:285-304.

90. Mellentin Al, Skot L, Nielsen B, Schippers GM, Nielsen AS, Stenager E, et al. Cue exposure therapy for the treatment of alcohol use disorders: a meta-analytic review. Clin Psychol Rev. 2017;57:195-207.

91. Verdejo-Garcia A, Clark L, Verdejo-Roman J, Albein-Urios N, Martinez-Gonzalez JM, Gutierrez B, et al. Neural substrates of cognitive flexibility in cocaine and gambling addictions. Br J Psychiatry. 2015;207:158-64.

92. Shafran R, Wroe A, Nagra S, Pissaridou E, Coughtrey A. Cognitive behaviour treatment of co-occurring depression and generalised anxiety in routine clinical practice. PLoS ONE. 2018;13:e0201226.

93. Carroll KM. Lost in translation? Moving contingency management and cognitive behavioral therapy into clinical practice. Ann N Y Acad Sci. 2014;1327:94-111.

94. Carroll KM, Ball SA, Martino S, Nich C, Babuscio TA, Nuro KF, et al. Computerassisted delivery of cognitive-behavioral therapy for addiction: a randomized trial of CBT4CBT. Am J Psychiatry. 2008;165:881-8.

95. Carroll KM, Kiluk BD, Nich C, Gordon MA, Portnoy GA, Marino DR, et al. Computer-assisted delivery of cognitive-behavioral therapy: efficacy and durability of CBT4CBT among cocaine-dependent individuals maintained on methadone. Am J Psychiatry. 2014;171:436-44.

96. Lee JL, Di Ciano P, Thomas KL, Everitt BJ. Disrupting reconsolidation of drug memories reduces cocaine-seeking behavior. Neuron. 2005;47:795-801.
97. Rhodes JS, Crabbe JC. Gene expression induced by drugs of abuse. Curr Opin Pharmacol. 2005;5:26-33.

98. Zhou Z, Enoch MA, Goldman D. Gene expression in the addicted brain. Int Rev Neurobiol. 2014;116:251-73.

99. Wang F, Liu Y, Guo S, Chen D, Sun H. The association between epoxide hydrolase genetic variant and effectiveness of nicotine replacement therapy in a Han Chinese population. Neurosci Bull. 2016;32:545-6.

100. Ducci F, Goldman D. The genetic basis of addictive disorders. Psychiatr Clin North Am. 2012;35:495-519.

101. Walker DM, Nestler EJ. Neuroepigenetics and addiction. Handb Clin Neurol. 2018;148:747-65.

102. Smith ACW, Kenny PJ. MicroRNAs regulate synaptic plasticity underlying drug addiction. Genes Brain Behav. 2018;17:e12424.

103. Jia X, Wang F, Han Y, Geng X, Li M, Shi Y, et al. miR-137 and miR-491 negatively regulate dopamine transporter expression and function in neural cells. Neurosci Bull. 2016;32:512-22.

104. Swiech L, Heidenreich M, Banerjee A, Habib N, Li Y, Trombetta J, et al. In vivo interrogation of gene function in the mammalian brain using CRISPR-Cas9. Nat Biotechnol. 2015;33:102-6.

105. Lareau CA, Clement K, Hsu JY, Pattanayak V, Joung JK, Aryee MJ, et al. Response to "Unexpected mutations after CRISPR-Cas9 editing in vivo". Nat Methods. 2018;15:238-9.

106. Dunbar CE, High KA, Joung JK, Kohn DB, Ozawa K, Sadelain M. Gene therapy comes of age. Science 2018;359:pii: eaan4672.

107. Chen $\mathrm{YH}$, Chen $\mathrm{CH}$. The development of antibody-based immunotherapy for methamphetamine abuse: immunization, and virus-mediated gene transfer approaches. Curr Gene Ther. 2013;13:39-50.

108. Anker JJ, Brimijoin S, Gao Y, Geng L, Zlebnik NE, Parks RJ, et al. Cocaine hydrolase encoded in viral vector blocks the reinstatement of cocaine seeking in rats for 6 months. Biol Psychiatry. 2012;71:700-5.

109. Murthy V, Gao Y, Geng L, LeBrasseur NK, White TA, Parks RJ, et al. Physiologic and metabolic safety of butyrylcholinesterase gene therapy in mice. Vaccine. 2014;32:4155-62. 